\title{
La justicia gratuita, entre el antiguo régimen y el sistema liberal constitucional
}

Free justice, between the ancient regime and liberal constitutional system

\author{
Dr. Jesús María Navalpotro Sánchez-Peinado, PhD \\ Docente titular de la Universidad de las Américas \\ Documento Original (Recensión) ${ }^{87}$ \\ RFJ, No. 3, 2018, pp. 425-435, ISSN 2588-0837
}

PALABRAS CLAVE: pobreza, abogados de pobres, defensoría pública, costas procesales, caso de Corte, derecho a la justicia.

KEY WORDS: powerty, poor man's lawyers, free justice, public defender, court costs, case of Court, right to justice.

Recensión de Bádenas Zamora, Antonio, Los litigantes miserables ante la Justicia de Fernando VII, Editorial Dykinson, Madrid, 2018, 145 pp. ISBN 978-84-9148-797-5

Review of Bádenas Zamora, Antonio, Los litigantes miserables ante la Justicia de Fernando VII, Editorial Dykinson, Madrid, 2018, 145 pp. ISBN 978-84-9148-797-5

\section{INTRODUCCIÓN: LA INVESTIGACIÓN Y SU AUTOR}

En ocasiones, al pasear por la Historia Jurídica, uno puede sorprenderse que haya avances que hoy se consideran indiscutibles, en su momento padecieron de retrocesos, justo al punto en que las proclamas liberales parecían establecer un mundo en el que el individuo fuera el centro de la vida política, económica y social. Antonio Bádenas, ya desde su primer gran trabajo de investigación, apuntó a las azarosas condiciones en que los que antes habían sido súbditos y ahora, fundamentalmente, eran ciudadanos, en algún aspecto, con la proclamación de las libertades individuales, quedaron en una situación de mayor

87 El presente documento fue sometido a revisión de pares externos 
desprotección que la que gozaban bajo el amparo de la justicia real del Antiguo Régimen.

La institución que se estudia en estas páginas, como acierta a poner de relieve el autor, inicialmente se contempló dentro de las funciones tuitivas del soberano sobre determinados sectores de súbditos, ya que, por motivos humanitarios, religiosos y caritativos, realmente todos ellos entreverados, la protección del débil se consideraba una exigencia para el gobernante cristiano. Habrá de ser el avance del pensamiento constitucional en el siglo XX el que inserte a la justicia gratuita entre los derechos de protección, exigibles a un Estado garantista: sin ella no es posible la verdadera justicia, que trate a todos por igual, como se reclama del poder público (p. 17). Ese es el cauce por el que lo podemos ver recogido en las declaraciones constitucionales de derechos de modo generalizado a partir de mediados del siglo XX.

Antes de proseguir, convendrá advertir elementos de interés para el lector ecuatoriano. Uno de ellos es de carácter general, de la historia política contemporánea, y consiste en percibir la paradójica y triste situación a la que el régimen liberal arrojó a los más desfavorecidos, en cuanto a su posibilidad de acceder a la justicia. La pretensión de igualdad original del liberalismo repelía los tratamientos desiguales. Eso, en relación con el acceso a la justicia, significaría cierta desprotección de los menos capacitados económicamente, que fue (y sigue siendo, en sus versiones más o menos evolucionadas en el siglo XXI) la asignatura pendiente de la ideología entonces triunfante. Otro elemento de interés es de Derecho Comparado: la justicia gratuita se halla constitucionalizada muy recientemente en España, como Bádenas recuerda, pues solo a partir de 1978, "el disfrute de la justicia gratuita dejó definitivamente de ser un privilegio y pasó a ser considerado un derecho público, de carácter subjetivo, habilitador del acceso jurisdiccional”. En Ecuador ocurre igual.

El Art. 75 del texto de 2008 así lo estableció, pero operando por distinta vía. En consonancia con declaraciones internacionales de derechos, se reconoce que "toda persona tiene derecho al acceso gratuito a la justicia y a la tutela efectiva, imparcial y expedita de sus derechos e intereses, [...]; en ningún caso quedará en indefensión”, y ello se completa con la creación de un órgano específico para "garantizar el pleno e igual acceso a la justicia de las personas que, por su estado de indefensión o condición económica, social o cultural, no puedan contratar los servicios de defensa legal para la protección de sus derechos" (Art. 191): la Defensoría Pública. En coordinación con ella, el art. 193, pre- 
vé servicios de defensa y asesoría jurídica a personas sin suficientes recursos y a grupos considerados "de atención prioritaria", por parte de las facultades de Derecho de las universidades (Defensoría Pública del Ecuador, web). También es práctica más o menos frecuente en los despachos las actuaciones, pro bono, sin contraprestaciones económicas, en favor de personas sin recursos. A lo largo de esta obra, el Dr. Bádenas desarrolla su investigación en un paso más acerca de la justicia gratuita, que inició con su propia tesis doctoral, galardonada con el premio extraordinario de Doctorado, acerca de sus avatares en los comienzos del Estado constitucional en España, bajo las regencias y el reinado personal de Isabel II. El trabajo de análisis jurídico que realiza con las fuentes históricas en este caso se ocupa de la época política anterior, los gobiernos de Fernando VII.

Convendrá hacer notar que el autor, ciertamente, posee dilatada experiencia como abogado, como profesor en las aulas universitarias y como minucioso y sólido investigador. En efecto, Antonio Bádenas Zamora se licenció en Derecho por la Universidad Complutense, donde se formó como investigador, aunque finalmente leyó su tesis doctoral en la Universidad Rey Juan Carlos, que fue distinguida con el Premio Extraordinario de Doctorado. Se inició en la docencia en esta institución universitaria, cuando comenzaba su andadura el Centro "Ramón Carande", que fue el germen de esa universidad. Heredero, académicamente, de una larga tradición de historiadores del Derecho, que desciende del magisterio de Alfonso García-Gallo, sus trabajos destacan el carácter jurídico de la disciplina y, de acuerdo con las líneas de la escuela, siempre ha cultivado la perspectiva institucional. Estudioso de la época contemporánea, especialmente, esta obra obedece a un proyecto nacional de investigación de las fuentes jurídicas del siglo XIX, acerca de "Las influencias extranjeras en la codificación penal española; su concreto alcance en la Parte Especial de los códigos decimonónicos".

\section{DESARROLLO: ESTRUCTURA Y ELEMENTOS RELEVAN- TES DEL LIBRO}

El autor propone, para entrar en su investigación, dos elocuentes citas: de un parlamento del ilustre jurista Lázaro de Dou ante las Cortes de Cádiz en 1811, y un texto de Bravo Murillo de 1836, en las que se pone de relieve la necesidad de facilitar a todos el acceso a los tribunales, superando para ello las barreras que puede imponer la falta de medios. Y desglosa su trabajo en seis capítulos, a los que siguen bibliografía y apéndice documental. 
La estructura está primorosamente trabajada, al punto que, puede considerarse un modelo de investigación histórico-jurídica: inicia con una breve y orientadora introducción ("Limitación del tema") en la que da cuenta del marco histórico y del estado de la cuestión, apoyándose en los conocimientos que a Bádenas le avalan como quizá el más experto conocedor en España del instituto de la justicia gratuita. Hijo de su tiempo, el autor reconoce desde el principio la visión negativa con que la historiografía ha retratado a Fernando VII y su reinado. Por extensión, también al Antiguo Régimen, del que este rey puede considerarse, con salvedades y matizaciones, como el último representante en el trono español. Pero, como la justicia y la honradez intelectual exigen al investigador, desde el principio, el autor ha de reconocer su sorpresa: aquel sistema político ofrece un saldo más beneficioso para el pobre que el que la ideología liberal propuso.

El segundo capítulo se ocupa del condicionante económico de la justicia a principios del siglo XIX. Es decir, qué elementos debían afectarse en la consideración de la gratuidad. Se desglosan y detallan los gastos de defensa o pagos a letrados y procurador (cuya intervención siempre era obligatoria), aranceles judiciales, papel sellado y depósitos para los recursos. Todos ellos tienen protagonismo en los debates posteriores. Unos costes que no excedían el ámbito privado, en el primer caso, y que solo eran temporales, por tratarse de una garantía, en el caso de los depósitos. Y dos tipos de tasas que servían a la Hacienda y al pago del personal de la administración de Justicia.

El siguiente capítulo se dedica a identificar a los beneficiarios de la gratuidad, lo que lleva a un estudio de los intrincados privilegios que, desde la Edad Media, habían ido favoreciendo a distintos grupos, equiparándolos a los pobres, de modo que por "miserables" se entendía, en una aplicación extensiva de un texto del Corpus Iuris Civilis, a todos los que "la injuria de la fortuna" les había llevado no solo a la pobreza, sino a la enfermedad, invalidez, viudedad, orfandad... (p. 36) Este fue uno de los grandes argumentos contra el instituto de la justicia gratuidad: su extensión y desvirtuación. El privilegio de litigar gratis se unía a otro de carácter jurisdiccional: el tratamiento como caso de Corte, casos que fueron aumentando con los años hasta constituir un número considerable, que les extraía a otras justicias y se llevaba ante los tribunales reales superiores. De estos efectos jurisdiccionales de la pobreza, y de otros más que se seguían, se ocupa el siguiente capítulo, 4, que, junto al 5 , expone la evolución del régimen jurídico de la gratuidad. Se desglosan los derechos que fueron reconfirmados: además de la consideración 
como caso de Corte, la representación y asistencia gratuita en juicio, la exención de aranceles, el uso de un papel sellado "de pobres", de precio muy reducido, y la exoneración de los depósitos para recursos.

Los casos de Corte son objeto de especial consideración; consistían, prácticamente, en que en determinados supuestos y a determinadas personas habían de ser los tribunales superiores (audiencias, chancillerías y sala de alcaldes de Casa y Corte) los que enjuiciarán en primera instancia. Refiere el autor que "la progresiva desnaturalización que con el paso del tiempo experimentó esta prerrogativa procesal, a causa fundamentalmente de haberse hecho extensiva a otras personas o instituciones procedentes de estamentos sociales superiores, justificó que los constituyentes de Cádiz concibieran la idea de eliminarla del ordenamiento jurídico" (p. 47). Y es lo que expone detalladamente en el siguiente capítulo. Bádenas no teme adentrarse en los volúmenes, a veces confusos, prolijos, de los debates parlamentarios. Una muestra de ellos la reproduce en su apéndice documental. Las Cortes de Cádiz decidieron suprimir el fuero privilegiado que constituían los casos de Corte. El objetivo liberal de acabar con fueros y desigualdades chocaba con la igualdad jurídica que se proclamaba. Pero, el autor da el título de "reconfirmación" a la situación jurídica posterior. Y es que, en efecto, el retorno del monarca "deseado" implicó la derogación de todas esas reformas procesales y la consecuente recuperación de los casos de Corte y de los privilegios jurisdiccionales a los "miserables". Además, en la España dominada por el "rey intruso" francés se habían aprobado reformas en la administración de Justicia, pero esta figura no había sido suprimida.

La asistencia gratuita en juicio no experimentó tampoco novedad. Constituía una carga que los letrados debían soportar "por amor de Dios". Desde tiempos medievales así se había entendido, que la asistencia era por cuenta del abogado, por cuyos servicios a los pobres nadie le satisfaría nada, excepto en unos casos en estaban designados que los concejos municipales. Lo que se había ordenado era un turno para que todos los abogados fueran atendiendo esas funciones (p. 54).

En cuanto a la exención de tasas, había sido objeto de reciente regulación en la Novísima Recopilación, en 1805, desarrollando asimismo disposiciones romanas y canónicas. Igualmente ocurrió con el "papel sellado de pobres", de precio notablemente inferior al que se exigía para los pleitos ordinarios, y la supresión de la caución para recurrir, que se mantuvieron tras la restauración fernandina. Una reforma en 1810 vino 
a eliminar un problema para los "pobres de solemnidad": se permitió que las actuaciones previas a su declaración fueran igualmente gratuitas, lo que había sido un a veces difícil obstáculo hasta el momento.

Los cambios se intentaron en torno al proceso, en general, y al establecimiento de los efectos de la declaración de pobreza, unidos al nuevo sistema de tasas. El autor sigue las transformaciones normativas y las que se frustraron en torno al proceso y a los sujetos que actuaban en él. Durante el reinado de Fernando VII no fueron efectivos los cambios, por las alteradas circunstancias en que no llegó a aplicarse la Constitución de Cádiz y, durante el trienio liberal, por la similar falta de tranquilidad política y social. Solo el cambio de reinado, y de régimen, en 1833, permitiría que las reformas se fueran implantando. ¿Cómo afectaban las modificaciones procesales al beneficio de pobre$\mathrm{za}$ ? Frente al sistema anterior, que confiaba al arbitrio judicial la concesión del mismo, se estableció un requisito objetivador, administrativo, que pasaba por la expedición de unos certificados municipales de pobreza, con ciertos requisitos. Así establecía el proyecto de código de 1821, que fue objeto de informes por distintas instituciones jurídicas: colegios de abogados, universidades y tribunales. En ellos se criticó que únicamente fuera la información tributaria la que sirviera de base a la consideración de pobre, lo que facilitaba el engaño.

Las conclusiones están expuestas en otro capítulo, el 6, bajo el rótulo de "Reflexión final". Sintetiza con precisión los cambios -mejoras- que el legislador realista (los gabinetes designados por Fernando VII) introdujo en la regulación del acceso gratuito a la justicia de los litigantes que, primero, dejaron de llamarse "pobres de solemnidad" o "miserables", y, en segundo lugar, se les precisó el modo de tasación para graduar la pobreza a efectos procesales, que los posteriores gobiernos liberales endurecieron, dejando fuera del beneficio a gran cantidad de familias realmente humildes.

Mención particular, entiendo, que merecen dos elementos que cierran la obra con un excelente broche: el apéndice documental y la bibliografía. El apéndice documental complementa, como en las obras clásicas de Historia del Derecho, el trabajo, mostrando el elemento de investigación histórica, con la ilustración mediante la exposición de las fuentes que han permitido realizar el análisis jurídico de la institución en su momento histórico. 
El Dr. Bádenas en este caso, incluye trece textos de acceso no siempre fácil, entre la maraña legislativa: primeramente, la Instrucción del Consejo de Estado de 1794 sobre papel sellado, aún en el ámbito de la Nueva Recopilación; una Resolución del Supremo Consejo de Guerra de 1799, difundida mediante Circular del Consejo de Castilla de 1800, tomada del Archivo Histórico Nacional; varios debates de las Cortes de Cádiz, de 1811, acerca de artículos del proyecto constitucional, alguna circular del Consejo de Castilla; informes de las universidades sobre los proyectos de codificación procesal,... En total, el investigador ha expurgado antiguas colecciones legislativas publicadas, la Gaceta de Madrid, así como el Archivo Histórico Nacional y el de la Comisión General de Codificación, lo que implica un trabajo minucioso y preciso, que, además, ha sido el que ha caracterizado sus anteriores publicaciones. En esta sección, lo que se echa en falta es un índice del apéndice documental, que facilite la consulta y permita una revisión conjunta de la documentación adjunta.

En perfecta serie, tras los documentos editados, se añade la relación bibliográfica. Ordenada de forma clásica, muy clara, distinguiendo entre literatura y diferentes tipos de fuentes jurídicas. Precisa con claridad encomiable los abundantes textos prelegislativos consultados, consistentes en debates parlamentarios, proyectos e informes institucionales para las reformas legales. Las docenas de fuentes normativas en las que se ha sumergido no se han limitado a la legislación general, a los grandes cuerpos legales de la Monarquía Española a finales del Antiguo Régimen, y a las posteriores que los sustituyeron, sino a las muchas que constan de carácter menor, administrativas, como circulares de los Consejos, y de órganos gubernativos inferiores posteriores (la Dirección General de Rentas, por ejemplo), que han permitido concretar la situación y aplicación del Derecho. Y la literatura consultada abarca toda la doctrina principal de finales del XVIII e inicios del siglo XIX. La brillantez de la obra se debe, en gran medida, a la exhaustividad de sus consultas.

A continuación, se ofrece la larga serie bibliográfica consultada, actualizada, y con atención a obras principales. Solo se me ocurre que podría haberse desplegado una visión más comparatista, con algunas consultas de obras paralelas en la literatura histórico-jurídica francesa, por ejemplo, o europea, en general, quizá hispanoamericana, que, aunque no pueda considerarse propiamente una carencia, sí que hoy en día es un elemento enriquecedor desde una perspectiva globalizadora. 
Lo indicado sobre fuentes y bibliografía se relaciona con la abundancia de aparato crítico, con una citación nuevamente minuciosa, prolija y bien detallada, en la que hay que agradecer que el autor no haya sucumbido a la desgraciada "dictadura" del sistema de citación APA, tantas veces manifestación (aún impulsada por algunas instancias del dirigismo oficial académico) de un papanatismo uniformador y distorsionador de la investigación científica. Cada afirmación, cada paso queda sólidamente sustentado en unas notas a pie de página pertinentes y eficaces. Que la editorial, en este caso, se haya prestado a hacer ese esfuerzo, es digno de agradecer, puesto que muchas veces son exigencias económicas y de comodidad del editor las que exigen un sistema más "actual" que, sin mayor justificación, simplemente relega el número y volumen de las aclaraciones al pie.

Y de la edición, también hay que mencionar que en este caso la editorial madrileña Dykinson, bien conocida en el ámbito jurídico, ofrece en este caso una tipografía clara y fácil de leer, con una portada original, tomada de un grabado con la efigie del monarca cuyo reinado enmarca el estudio.

\section{CONCLUSIONES FINALES, DESDE AMÉRICA}

1. En primer lugar, hay que destacar que la obra escrita por el profesor Bádenas, tiene interés para el jurista, para el historiador y me atrevería, sin exageración, a reiterar otra consideración anterior: cabe considerarla como un modelo de investigación histórico jurídica (en especial en nuestro país, tan maltratado por los "modelos" oficiales de investigación, que la reducen a elementos formales y cuantitativos, a serie de citas a cuento o no). Se puede considerar que, a partir de un instituto jurídico concreto, la justicia gratuita, aparecen elementos pluridisciplinares, dentro del estudio de la Jurisprudencia: Derecho Constitucional, Derechos Humanos, Derecho Procesal, Derecho Tributario, además de la Historia de las Ideas Políticas.

2. Aunque no hay referencias expresas a América, realmente el trabajo es también de interés en nuestro continente. El tiempo del estudio, es el tiempo de las luchas por la independencia y, entre tanto, las reformas en la Administración de Justicia en España, teóricamente también habrían de ser aplicables en los aún denominados reinos de Indias. Con carácter general, la legislación menos afectada por el cambio político (esta, la constitucional y, en parte, la penal) fue la civil y 
procesal, y se continuó aplicando en las nuevas repúblicas, hasta que nuevas leyes al cabo de los años, no pocas veces de las décadas, fueran supliendo al Derecho Indiano por los derechos nacionales.

Las Cortes de Cádiz, desde luego, tuvieron presente a los reinos americanos españoles, con unas pretensiones de igualdad más discursivas que reales, conforme expresaba el decreto de 22 de enero de 1809 , despachado por la Junta Central en España: "los vastos y preciosos dominios que España posee en las Indias no son propiamente colonias o factorías como las de otras naciones, sino una parte esencial e integrante de la monarquía española" (Pereyra, 1989, p. 394).

Sin embargo, la coyuntura histórica se podría sospechar que no favoreciera la llegada de las pequeñas reformas sobre justicia gratuita hasta la Real Audiencia de Quito. Desde 1810 había comenzado un proceso de separación del gobierno de España, aunque fuera bajo el mismo rey, Fernando VII, ausente, con resultados inicialmente frustrados. Pero luego transcurrió la siguiente década de guerras civiles, que acabaría con la separación de los territorios americanos, parte de ellos agrupados en lo que después se conocerá como la Gran Colombia. Larrea hacía notar que, aunque entre 1809 y 1822, "en el país no se produce otra obra de creación jurídica, fuera de él, en las Cortes de Cádiz y en Colombia, se legisla y estos instrumentos legales tendrán vigencia en el futuro Estado o influencia en su vida legal" (Reig \& Larrea, 2000, p. 221).

3. En cualquier caso, desde el Derecho Comparado y desde la Historia del Pensamiento Político esta breve investigación es dilucidadora. En ella se refleja el modelo tradicional de amparar a los desfavorecidos económicamente, y a otros muchos que eran equiparados con ellos presentaba como rasgos más problemáticos la dependencia del arbitrio judicial y el cargo de los costes de representación sobre los propios letrados. Pero parece que resultaba más beneficioso como garantía del acceso de todos a la justicia. Fernando VII y sus gobiernos realistas mantuvieron ese modelo. En cambio, el liberalismo primeramente intentó eliminar algunos de los efectos que tenía la declaración de pobreza, como los casos de Corte, hizo más rigurosos los requisitos para beneficiarse de ella y, en realidad, obedeció a un criterio contrario a su pervivencia, por atentar contra el axioma político de la igualdad de los ciudadanos. 
Y frente a ese modelo tradicional, el liberal. Excede de las páginas del libro presentar cómo se desarrolló este en otros lugares (por ejemplo, los estudios clásicos para Gran Bretaña de Abel-Smith \& Stevens, 1967, 1968). No era el objeto de estudio. Aunque habría ilustrado el contexto ofrecer una pincelada de cómo se trató la garantía del libre acceso a la justicia en otros sistemas. El modelo liberal más puro será el que pretenda que sean consultorías jurídicas gratuitas, y las actuaciones por bono, las que cubran las carencias de aquellos que carecen de medios económicos. En definitiva, dejan el acceso a la justicia de los desfavorecidos, al albur del altruismo o generosidad de un despacho profesional, con los problemas que se derivan de lo que pueda ocurrir en poblaciones pequeñas o en casos complejos que implicarían escaso prestigio y gran empleo de tiempo. Desde luego, ese modelo no se corresponde plenamente con la idea de un derecho humano de acceso a la justicia en igualdad de condiciones. En un salto en el tiempo, se puede recordar cómo en Ecuador, en otro extremo, se ha optado por un modelo fuertemente estatalizado, coherente con los planteamientos intervencionistas gubernamentales que se expresaron en la Constitución de 2008. Antes existían defensores de oficio (en 2007 eran una treintena), pero la realidad era que miles de presos se hallaban sin haber sido sentenciados (Defensoría Pública del Ecuador, web). El nuevo sistema depende enteramente del Estado.

4. Del libro se destaca, pues, su interés, primero, por la metodología: como análisis jurídico riguroso, con una dogmática bien construida y explicaciones muy claras, que hacen gala de un excelente uso de la lengua; y, en segundo lugar, por su propio contenido, innovador y enjundioso para la Historia del Derecho.

\section{REFERENCIAS BIBLIOGRÁFICAS}

Abel-Smith, B. and Stevens, R. (1968). In Search of Justice: Law, Society and the Legal System. Londres: Allen Lane The Penguin Press.

Abel-Smith B. and Stevens, R. (1967). Lawyers and Courts. Londres: Heinemann

Defensoría Pública del Ecuador [página web]. Reseña histórica. Disponible en: http://www.defensoria.gob.ec/index.php/defensoria-pu- 
blica/quienes-somos/resena-historica Pereyra, Carlos (1989). Breve Historia de América. México: Editorial Patria, $5^{\mathrm{a}}$ ed.

Reig Satorres, J. y Larrea Holguín, J. (2000). Manual de Historia del Derecho en el Ecuador. Quito: Corporación de Estudios y Publicaciones.

Recibido: 18 de agosto de 2018

Aceptado: 6 de noviembre de 2018

Dr. Jesús María Navalpotro Sánchez-Peinado, PhD: Docente titular de la Universidad de las Américas

Correo electrónico: jesusmaria.navalpotro@gmail.com 